Research Article

\title{
Teaching Mode of Sports Swimming Video in Colleges and Universities Based on Artificial Intelligence
}

\author{
Jun Ma \\ Sports Department, Guizhou University of Finance and Economics, Guiyang 550025, Guizhou, China \\ Correspondence should be addressed to Jun Ma; majun@mail.gufe.edu.cn
}

Received 19 June 2021; Revised 12 July 2021; Accepted 31 July 2021; Published 17 August 2021

Academic Editor: Sang-Bing Tsai

Copyright (c) 2021 Jun Ma. This is an open access article distributed under the Creative Commons Attribution License, which permits unrestricted use, distribution, and reproduction in any medium, provided the original work is properly cited.

\begin{abstract}
After mankind entered the 20th century, a young science with a history of only 60 years, artificial intelligence technology, is affecting the world in its own unique way. Artificial intelligence technology is known as one of the three major scientific and technological achievements of this century. The applications of artificial intelligence technology include machine learning, expert systems, pattern recognition, natural language processing, artificial neural networks, and robots. Two classes $(n=42)$ were randomly selected in the experiment, namely, the experimental group and the control group. The control group adopts traditional methods of teacher explanation, demonstration, and practice. The experimental group uses a combination of feedback and traditional teaching methods to teach. The students in the experimental group and the control group are compared in the early and late stages of the swimming skills video. Finally, the characteristic parameters of swimming techniques are compared and analyzed. Experimental data showed that the average score of students in the experimental group was 32.38, and the average score of the control group was 28.43. The average technical evaluation score of the experimental group was 77.19 , and the average technical evaluation score of the control group was 72.43. The experimental results show that the video feedback teaching method does play a certain role in improving students' technical movements and proficiency and improves the quality of teaching.
\end{abstract}

\section{Introduction}

Since it was first proposed at the Dartmouth Conference, artificial intelligence has experienced a freezing period like the development of other disciplines, and now, it is developing rapidly due to the rapid development of brain and cognitive disciplines. Artificial intelligence is known as one of the three major scientific and technological achievements of this century. Among them, the application of artificial intelligence technology, such as machine learning, has spawned many new positions and product applications. Talents who master the application of artificial intelligence technology have become the "fragrance" of major IT companies. Lu Qi's entry into Baidu and Li Feifei's entry into Google have brought a lot of social attention. The application of artificial intelligence technology, such as machine learning, expert systems, artificial neural networks, and robots, has opened up new markets, bringing many people to important fields such as medical diagnosis, transportation, education, criminal justice, finance, environment, and energy.

Sports is different from other fields in that it relies on body language. Students must establish the correct impression of actions in the brain through observation and perception and use the inherent sensations of muscles to perceive the power of various technical actions, spatial characteristics, and time of actions. Video education mode can stimulate students' enthusiasm for learning and help broaden their horizons. Video teaching is more dynamic, novel, and diverse than traditional education technology and can arouse students' enthusiasm for learning.

With the rapid development of computer technology and the progress of multimedia communication technology, various industries have conducted in-depth research and application in video research. Sakamoto believes that, in some surgical fields, hands-on learning of benchmark models may be more effective than traditional teaching practices. However, microsurgery has not been reported. His 
research goal is to prove the effectiveness of hands-on learning of benchmark models in acquiring microsuturing skills. The secondary goal is to assess the ability of microsurgery based on personality assessment. He randomly assigned 86 medical students, including 62 men and 24 women, to 20 minutes of hands-on learning using a desktop model simulator or 20 minutes of video learning using instructional videos. The final product was scored blindly by two independent raters. Then, he conducted a personality test on all participants and compared their microsuture test scores and time to complete the task. Although his research has certain reference value, it has no innovative significance [1]. Yao believes that functional dependency and association rules are two important concepts in database systems, and they are also difficult points in the teaching of database system introductory courses. Functional dependence is the theoretical basis for the standardization of relational patterns, which reflects a restricted relationship between attributes in the relationship. Association rule mining is an important research content of data analysis. He makes full use of the relationship between the itemsets implicit in the functional dependency. Although his research is theoretically correct, it lacks experimental data [2]. Peng believes that the widespread use of the Internet and Internet-based devices allows people to easily access big data. With the emergence of big data, the traditional teacher-centered teaching model can no longer arouse students' interest in learning, nor can it meet their overall progress needs. He proposed an innovative teaching model for teachers and students (BEST) based on a big data environment to improve teachers' teaching effects and students' learning efficiency of big data. He analyzed the challenges brought by big data and proposed the corresponding best teaching model based on big data [3]. Hu et al. proposed two methods based on data envelopment analysis (DEA) and PageRank to rank college sports coaches [4]. However, by analyzing the challenging problems in the big data reaction, his research data volume is not large and the basis is insufficient.

This paper realizes the integration of video teaching and physical education teaching and provides more information technology support for swimming teaching. The use of video teaching can deepen educational thinking, enrich educational content, promote educational reform, realize diversification, subjectivity, and socialization, and comprehensively improve the quality of education are of great significance. Through video explanation, teachers can combine slow motion, settlement, regeneration, and other educational methods as explanations that are difficult for them to demonstrate, allowing students to see a more comprehensive image of actions, emphasizing key actions, improving educational effects, and shortening the educational process.

\section{Mode of College Sports Swimming Video Teaching}

2.1. VR Technology. VR is based on computer simulation technology as the core, and the three-dimensional space design and planning in the computer will eventually generate a virtual reality world. The user uses a professional input tool or output tool environment, and the user can find things in the three-dimensional space without space restrictions and finally achieve the goal of close to the real e-world experience and convenient and natural humancomputer interaction. The core feature of VR is immersive. Immersion means that the user can be immersed in the virtual environment generated by the computer system, and the virtual objects in the virtual environment can perform autonomous activities in the virtual environment. Use sound to observe and observe all content in the virtual environment with special equipment, not just stay on the external audiovisual computer of the computer or directly monitor the processing results of the computer. Interactivity refers to the feeling that the user is no longer passive in the virtual environment. The user's operation of information is no longer limited to using the mouse or keyboard, but objects such as data sensors, which are used to interact with objects in the virtual environment. Imagination emphasizes that VR has a wide range of imagination and can expand the scope of human brain knowledge. VR cannot only realize the existence of a real environment but also allow users to give full play to their imagination. In other words, users can freely construct situations that are completely nonobjective or even nonexistent [5].

VR is to help users enter a virtual environment through a head-mounted display device for an immersive interactive experience. The environment created by VR is virtual. AR is developed on the basis of VR. On the basis of the real environment, virtual digital images are produced by computers so that the real environment can be enhanced. VR emphasizes the use of computer technology and panoramic images to provide users with a virtual three-dimensional environment with a strong sense of reality, while AR must combine images and sense of reality to enhance the sense of reality. AR creates a real environment that combines virtual and reality. Different characteristics produce different experiences and positioning. VR is more suitable for entertainment fields such as games and film and television because they are more suitable for a virtual environment that can realize immersive experience. VR will make people more and more immersed in the virtual environment, while AR will make people walk into reality [6].

2.2. Video Teaching. Most people have not been exposed to the virtual environment, and the inadaptability during the training and the process will cause the error of the experimental data, which is caused by random. To capture a video, of course you need a video input source. Analog TV signals, analog video cameras, digital video cameras (DV), and old cassette video recorders are common video input sources, among which TV and DV are the main collection objects. The former signal is analog, and the latter is digital. Before the analog/digital conversion, what needs to be determined is the acquisition frequency, that is, how many frames are acquired in one second. If the set number of acquisition frames is high, the generated video file will be smoother, but the acquisition speed will be slower; on the contrary, if the 
set number of acquisition frames is too low, the video image may not be coherent. The eyes are quite uncomfortable, but its collection speed is fast [7].

The teaching process is a two-way activity, involving the mutual participation between teachers and students and the communication process between people. Video feedback teaching is interactive. The teaching content of video feedback teaching is not only the theoretical knowledge taught in traditional teaching but also the emotional communication between teachers and students in the teaching process. The emotional communication between teachers and students is more conducive to the development of teaching activities. The emotional communication between teachers and students helps to establish a relaxed and pleasant environment. It is the prerequisite for enhancing teaching ability and the basis for teachers and students to complete the teaching goals together. It can activate students' thinking, enhance students' memory, and arouse students' learning autonomy [8]. The video feedback teaching method combines sight, hearing, appearance, and other senses, allowing students to better grasp the specific details of difficult behavior in the form of slow motion, understand video feedback information more intuitively, correct wrong behavior in time, and can avoid accidents, cultivate good teaching habits, and effectively improve teaching efficiency. Video feedback teaching methods can timely feedback information, correct errors, avoid the accumulation of errors as much as possible, overcome the formation of directional thinking and habits, and train students to understand the concepts, principles, and laws of corresponding knowledge in the way of coding procedures in the brain, and rehearse to a certain degree The structure of the video media can consolidate the knowledge learned and, at the same time, cultivate practical skills while learning theories, and one can use the dynamic form of video media to better understand and master abstract concepts. When we just get the amount of information that is unmatched in previous video viewing by just watching, the image effect of the VR video will get a depth that cannot be achieved in previous video viewing. What you see is what you think. The picture elements and picture clips seen in previous videos all contain the creator's own intentions. Your thinking about the picture is also largely driven by the creator's thinking. The resulting thoughts are inevitably influenced by other factors. The VR video generated by virtual reality technology uses a lens-bonded screen generation method to form a space for the audience to form a screen image. Such a space completely liberates the limitations of the thinking space caused by the previous single screen switching. On the screen in the virtual reality space, you and I see more, and the amount of information used to form independent thinking is richer. Furthermore, because everyone focuses on space differently, the spatial information they are exposed to must be different, so after each person is exposed to the VR video, their focus will be different, and the result of accepting a VR video is also different from person to person $[9,10]$.

2.3. Video Image Processing. The digital camera takes the scene to obtain the image, which is completed by the principle of optical imaging. Four coordinate systems are needed in the whole process. When the object is photographed, it needs to be converted to the tangent plane of the spherical surface with the camera focal length as the radius, which is the camera coordinate system at this time. When observing an object from an image, the coordinate system of the object at this time is the image coordinate system. Finally, when starting to study the image matrix by pixel, the coordinate system has become a pixel coordinate system. The whole process of optical imaging is actually the conversion process of these four coordinate systems [11].

Gray information is the pixel value of the image, and it is the most direct way to register using the relationship of the pixel value of the image. In this method, the pixel value of each row (or column) is compared with the row (or column) pixel value of other images in the current image sequence to be stitched until the pixel value closest to the current row (or column) is found. By analogy, after traversing all row (or column) pixel values, find an image that is closest to its own pixel value as the adjacent image. In this registration method, the algorithm execution process is simple, but the amount of calculation is large, not the best choice for image registration [12].

Calculate the difference between the reprojection coordinates of the calibration template and the actual value $m_{i j}$ in $3 \mathrm{D}$ space and set up the error model as follows:

$$
r=\sum_{i=1}^{n} \sum_{j=1}^{m}\left\|m_{i j}-\widehat{m}\left(A, R_{i}, t_{i}, M_{i}\right)\right\| .
$$

When the original image is input, the shift algorithm first uses the Gaussian kernel to scale the image, and the formula is as follows:

$$
G(x, y, \sigma)=\frac{1}{2 \pi \sigma^{2}} \exp \left(\frac{-\left(x^{2}+y^{2}\right)}{2 \sigma^{2}}\right) .
$$

Statistics are classified according to the gradient direction of each pixel, the direction range is $[0,36]$ degrees, every 10 degrees is a group, and the peak value in the histogram statistics is used as the main direction of the current feature point. After determining the main direction, in order to ensure the rotation invariance of the algorithm, the coordinate axis is rotated by $\theta$ degrees to make it consistent with the main direction. The gradient value and direction of the new coordinate of the pixel in the field after rotation are, respectively, 


$$
\begin{aligned}
& m(x, y)=\sqrt{(L(x+1, y)-L(x-1, y))^{2}+(L(x, y+1)-L(x, y-1))^{2}}, \\
& \theta(x, y)=\tan ^{-1} \frac{(L(x, y+1)-L(x, y-1))}{(L(x+1, y)-L(x-1, y))} .
\end{aligned}
$$

2.4. Application of Video Teaching in Swimming. From the perspective of system theory, a comprehensive study of the theoretical system of swimming technology is carried out. It is believed that swimming is a complete system composed of four aspects: technical level, tactical level, psychological level, and physical level. This is an indivisible whole that is interrelated, interdependent, and mutually restricted. These four levels have their own connotations. In swimming teaching and sports practice, the viewpoint of system theory is used to comprehensively and profoundly understand swimming techniques and to conduct targeted training and update on each level of specific swimming techniques. The concept of swimming technique teaching and training deepens the understanding of the connotation of swimming technique, in order to guide the practice of swimming technique, improve the quality of technique in swimming competition, and obtain the best effect. From the perspective of the combination of biomechanics, anatomy, and psychology, a comparative study of athletes of different swimming skills is carried out to solve the problems in swimming teaching and training practice. From the perspective of sports culture, discuss the history, current situation, and development trend of swimming and discuss the problems in swimming teaching and training $[13,14]$.

\section{Sports Swimming Video Teaching Experiment}

3.1. Subject. Randomly select 2 swimming option administration classes; each class has 84 students, a total of 168 are experimental subjects. Through statistical analysis of the pretest data, it was confirmed that there was no significant difference in the general fitness of the selected three groups of test subjects. The difference does not matter; finally, one class in the experimental group is determined and 1 class in the control group is determined as 84 students each [15].

3.2. Teaching Methods. The experimental group uses video technology analysis, feedback, and traditional teaching methods, combining the early and late swimming technique teaching videos of the experimental group and the control group students, and finally compares the characteristic parameters of the swimming technique of the experimental group and the control group [16].

\subsection{Experimental Process}

(1) Before officially starting the experiment, in order to avoid the difference in physical fitness between the experimental class and the control class and occurrence of unnecessary errors in the experimental results of this experiment, before the start of the experiment, first check the experimental class and the control class. The students in the class took a pretest of their physical fitness. The physical fitness test content includes age, height, weight, half-meter reentry running, one-minute rope skipping, and front throwing a solid ball.

(2) The control group used the traditional teaching process focusing on skill training. The trainees passively accepted technical movements and practiced repeatedly. Second, conduct group exercises in accordance with the formulated unified education plan. Teachers make collective and individual corrections in the exercise. On the 8th and 20th day of training, the swimming movements of the students in the control group were photographed, and the first area and the second area were measured. Each region will have 10 success rates and standardized scores for behavior.

(3) The experimental group will use video and image technology to support the teaching methods of the traditional education model. The specific execution process is as follows. Teachers explain and demonstrate, configure students to watch slow motion playback of the world's leading swimmers, and help students establish correct expression perception. During the teaching process, the students' movements are tracked and the video is taken every day, and teaching tasks to improve the standardization of technical movements are formulated, and the students' technical movements that have been taken and processed are shown to the students at the beginning of the second day of training. In the training of the day, complete the teaching and training tasks to make the students' technical movements reach the standard. The teacher refers to the standard collective and individual error correction of athletes' swimming [17].

\section{Video Teaching Mode Analysis}

4.1. Analysis of Experimental Results. Swimming is a highly skilled sport with complex movements. The main technical movements are divided into breathing, foot movements, arm movements, foot movements, and breathing and whole body coordination. The movement of the feet and arms is the key point, while the coordination of breathing and the whole body is difficult. Pay attention to difficult learning directions, while speeding up the speed of students mastering this sport in actual training. The problem that this application design can solve is that, in the teaching process, when the mastery of the movement essentials is not comprehensive, the relevant theoretical knowledge and the movement essentials are not 
correct, and the purpose of solving the problem cannot be achieved through demonstration teaching, and the learning through the design research can solve the above problems. Students' interest and passion for swimming learning are also conducive to the development of the augmented reality swimming teaching mode [18]. The augmented reality interactive part of the virtual teaching platform is equipped with a 3D video tutorial display. With the virtual somatosensory experience, the teaching is more vivid and more acceptable to students, and it also deepens the understanding of swimming. Table 1 shows the physical fitness of the experimental class and the control class before the experiment. Among them, the test of height and weight is to verify whether there is a significant difference in the morphological indicators of the experimental class and the control class. After the independent sample $T$ test, it is found that the $P$ value of height is 0.75 , and the $P$ value of weight is 0.59 , both of which are greater than 0.05 , indicating that there is no significant difference in morphological indicators between the experimental class and the control class. The half-meter turn-back run is chosen to test the students' ability to change direction, quick reaction, quick start, and braking. Oneminute rope skipping is to test the students' hand-foot coordination and endurance. Throwing a solid ball forward is to test the student's explosive power and the ability of the body to coordinate and exert force. After the independent sample $T$ test, it was found that the $P$ value of the half-meter reentry running item was 0.25 , the $P$ value of the one-minute skipping item was 0.07 , the $P$ value of the front throwing solid ball item was 0.82 , and the $P$ value was also greater than 0.05 . Therefore, the above data shows that there is no significant difference in physical fitness between the experimental class and the control class, and their physical fitness levels are roughly the same, which meets the requirements of the experiment $[19,20]$.

The comparative analysis of the technical assessment results of the two groups of students is shown in Table 2 and Figure 1. The average score of students in the experimental group was 32.38, and the average score of the control group was 28.43. The average technical score of the experimental group was 77.19, and the average technical score of the control group was 72.43. In the second stage of the results, the standard deviation and technical evaluation results of the control group in the javelin were lower than the experimental group $P<0.05$, and there was a significant difference. It shows the application of VR panoramic video feedback in research. Motor skills have an impact on the prevention and correction of motor skills. The progress of the experimental group students is obvious. Cognitive theory believes that human learning is a process of external knowledge, which is internalized in the psychological structure and reorganizes knowledge based on original experience. Cognitive theory examines the importance of the cognitive structure in learning from the perspective of human psychological activities, emphasizing that people's learning process, mental process, or new skills are consistent with new information. Cognitive theory mainly has distributed individual cognition and interactive cognition. Individual cognition refers to the perception of the subject, that is, learning through relatively simple coding and memory, that is, the inner cognitive process of students. Interactive cognition in VR is an individual cognitive model that represents the subject's perception, including visual, auditory, and tactile perception. Distributed cognition is a system that includes cognition and environmental issues, and it is also an information processing process, that is, the processing of internal and external characteristic information. Cognitive activity is a kind of cognitive activity that emphasizes the cognitive distribution among individuals, individuals and cognitive media, cognitive environment, time, and society. Video collection based on motion monitoring is a continuous process. Through motion monitoring to control the storage and alarm of video data, the storage efficiency is greatly improved, and the storage space is effectively saved $[21,22]$.

4.2. Analysis of the Teaching Mode of Physical Education Video in Colleges. Through the analysis of static graphics, text, and annotation information, important information can be well marked, and a qualitative analysis of human movement can be made: through the analysis of key frames, the movement process can be well-refined and decomposed, and a summary of human movement information can be made. Through the comparative quantitative analysis of the twostage and three-stage window movement, an overall, comprehensive, and staged analysis of human movement can be made. All the information analysis and results displayed in the window can be saved in the current video file and exist with the video file in the form of dat, or can be processed in the current video file by copy, paste, cut, delete, and other functions. When the video file is opened again, the last analysis results are saved in the view, which is convenient for users to understand and reprocess the video information. The comparison of the experimental results of the three classification algorithms is shown in Figure 2. It can be seen from the figure that the TPR values of the three classification algorithms are all very high, indicating that the evaluation indicators of normal people identified by the classifier account for a high proportion of all positive instances; the FPR values of the three classification algorithms are all very small, indicating that the classifier mistakenly believes that abnormal index data in normal people account for a low proportion of all abnormal index data; the precision values of the three indicators are all high, indicating that the classifier has a high probability of correctly predicting normal people as normal people; the recall values of the three classification algorithms are all very high. In the original normal sample, the probability of being correctly predicted as a normal person is high; the $F$-score of the three classification algorithms is very high, indicating that the classifier is for normal people, and the classification effect is good; the Kappa values of the three classifiers are all close to 1 , indicating that the selected three classifiers are excellent. The application of motion analysis technology based on video acquisition largely depends on the effect of video acquisition. The control of the video stream in the acquisition, that is, the acquisition frame rate, affects the video definition and the playback speed in subsequent analysis. 
TABLE 1: Comparison of physical fitness between the experimental class and control class before the experiment.

\begin{tabular}{|c|c|c|c|c|}
\hline Test index & Group & $X \pm S$ & $T$ value & $P$ value \\
\hline \multirow{2}{*}{ Age } & Experimental class & 21 & 0.47 & 0.64 \\
\hline & Control class & 23 & 0.33 & 0.55 \\
\hline \multirow{2}{*}{ Height (m) } & Experimental class & 1.85 & 0.41 & 0.75 \\
\hline & Control class & 1.78 & 0.54 & 0.61 \\
\hline \multirow{2}{*}{ Weight (kg) } & Experimental class & 73 & 0.55 & 0.59 \\
\hline & Control class & 68 & 0.44 & 0.36 \\
\hline \multirow{2}{*}{ Half-meter turn-back run $(\mathrm{m} / \mathrm{s})$} & Experimental class & 17.52 & 1.16 & 0.25 \\
\hline & Control class & 18.11 & 1.55 & 0.78 \\
\hline \multirow{2}{*}{ One-minute skipping rope (times/min) } & Experimental class & 152 & 1.83 & 0.07 \\
\hline & Control class & 149 & 0.12 & 1.16 \\
\hline Toss a solid ball forward (m) & Experimental class & 9.87 & 0.23 & 0.82 \\
\hline Test index & Control class & 8.79 & 0.55 & 0.49 \\
\hline
\end{tabular}

TABLE 2: Comparison of the technical assessment results of the two groups of students.

\begin{tabular}{lcc}
\hline Group & Achievement & Technical evaluation results \\
\hline Experimental class & 32.38 & 77.19 \\
Control class & 28.43 & 72.43 \\
$T$ & 3.834 & 3.516 \\
$P$ & 0.015 & 0.021 \\
\hline
\end{tabular}

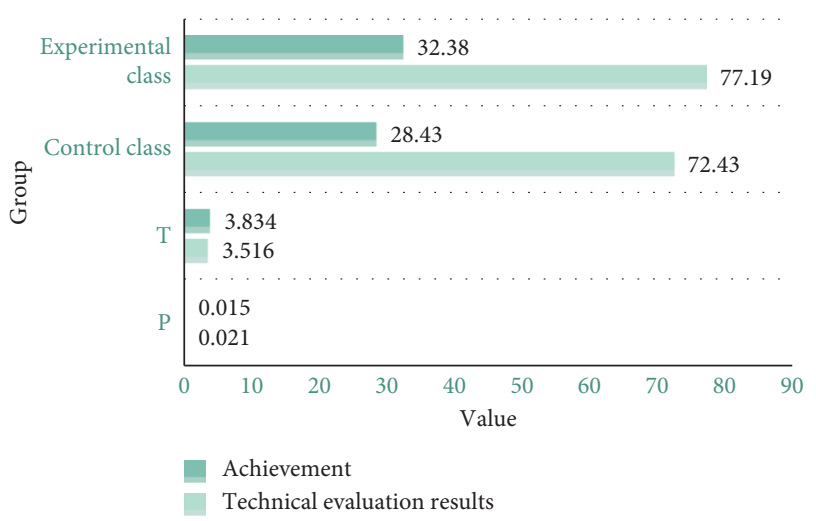

Figure 1: Comparison of the technical assessment results of the two groups of students.

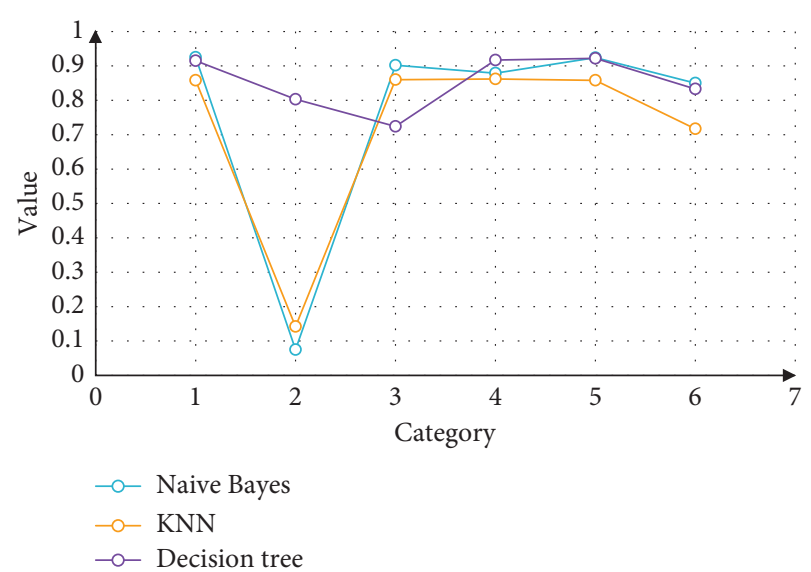

FIgURE 2: Comparison of experimental results of three classification algorithms.
Video program development adopts DirectShow technology in Microsoft Media Development Kit DirectX. The software module is directly included in the released Windows operating system. It can be upgraded as an independent module for free. DirectShow divides the video processing into objects with different functions by using a component object called filter and transfers direct and complex physical hardware operations to the underlying WDM driver. In terms of video sources, the video sources that DirectShow can handle can come from files, high-speed networks, and video capture cards, and the video stream data can be in different encoding formats. In the process of decompressing and compressing the media stream, the third party provides components to realize the decompression and compression algorithm. In the display part, DirectDraw and DirectSound technologies are used to efficiently output the data stream to the video card so that we can obtain professional video effects during development. In the storage, the video data can also be compressed and stored in a specified file in a certain format through functional components [23].

4.3. Application Analysis of Video Teaching Mode. The use of multimedia technology in the teaching of swimming courses, as a teaching aid, has the following functions. The first is to create a problem situation; the second is to clearly display the action structure so that students form a dynamic action representation; the third is to stimulate students' learning enthusiasm and interest in learning and to cultivate self-study ability; the fourth is to record and carry out the students' actions in time. Feedback, while comparing with correct actions, enables students to clearly discover their own mistakes and correct them in time. It changes the past because they cannot see their actions and can only perform actions through the teacher's explanation and their own proprioception. The video image technology teaching method has created a good teaching and learning environment. Through this kind of education method, students cannot only obtain a distinct learning environment but also enable teachers to have a high-level, high-quality educational environment. Therefore, teachers can implement humanitarian teaching methods, and students can explore, learn, and train creative thinking skills. Education and learning 
promote each other, making the boring educational process vivid and interesting. With the integration of educational information, educational content has become richer and the amount of information has also increased. Books, documents, videos, etc. are all cultural information materials. Any kind of information is just a part of the teaching material. Video and image technology will make the best use of media technology, fully collect all educational elements, greatly enrich the educational content, allow the educated to gain more knowledge in a short time, and improve the classroom and the efficiency of teaching [24]. Figure 3 shows the comparison of the results before and after the teaching experiment. It can be seen from the figure that the CPAVOD system is used for swimming teaching, and the teaching content is displayed in a unique form of video, while the swimming video presents the teaching content to the students in a complete and orderly manner. Students can fully analyze each set of swimming postures and standard actions, perceive their own deficiencies in learning, improve their swimming level in contrast to learning, and give full play to the main role in the learning process to achieve good teaching results. Traditional physical education teaching is entirely centered on teaching. Traditional physical education theory emphasizes that students are the subject of physical education, and everything is based on students. However, it is difficult to achieve this goal in actual teaching. In actual education, many teachers are blindly enlightened, and students will passively accept it. As time goes by, students' interest in learning will weaken, and their enthusiasm for learning will also be affected. In turn, students' attention in class will also be affected. As a result, the learning effect of movements is not good, and simple movements require a lot of time. Learning, complicated movements, or slightly difficult movements cannot be completed at all. This is the status quo of traditional teaching. The use of video teaching, through watching the video, improves students' interest, improves students' attention, makes students passive acceptance into active learning, and changes students' thinking, so as to better achieve teaching objectives [25].

The teacher's evaluation of the swimming video teaching experiment classroom is shown in Figure 4. In the classroom grading situation of the teachers in the two classes, the scores of 3 lessons in the experimental class were slightly higher than those of the control class, and the scores of 1 lesson were slightly lower than the control class. This shows that there is not much difference in the teaching situation of the two classes and that the teaching situation of the two classes is not much different in some indicators. The total average score of the four-class teacher evaluation of the two classes in the experimental class is slightly higher, which also shows the teachers' recognition of the swimming video teaching class. After observing teaching experiments, some teachers believe that the swimming video teaching classroom can achieve a certain teaching effect, so from the perspective of the recognition of the classroom to the swimming video teaching classroom, the development of swimming video teaching is worth exploring. In the early stage of the experiment, video image technology was not used to assist teaching, and there was no significant difference between the

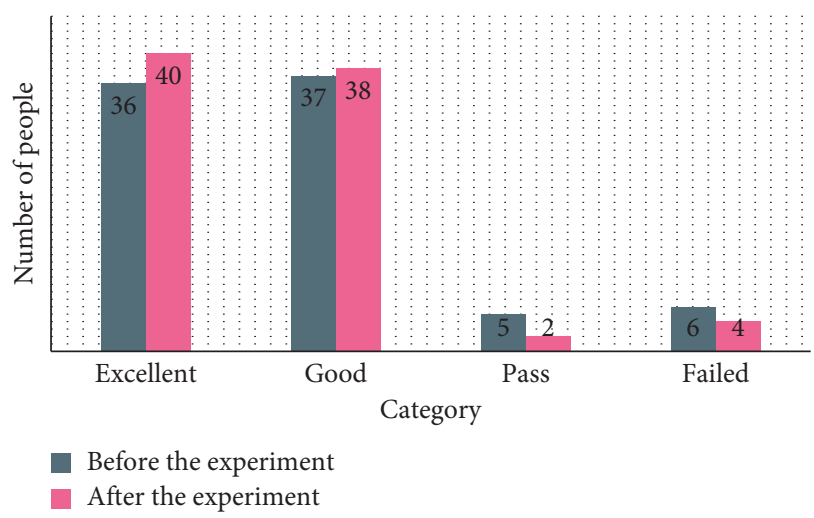

FIGURE 3: Comparison of results before and after teaching the experiment.

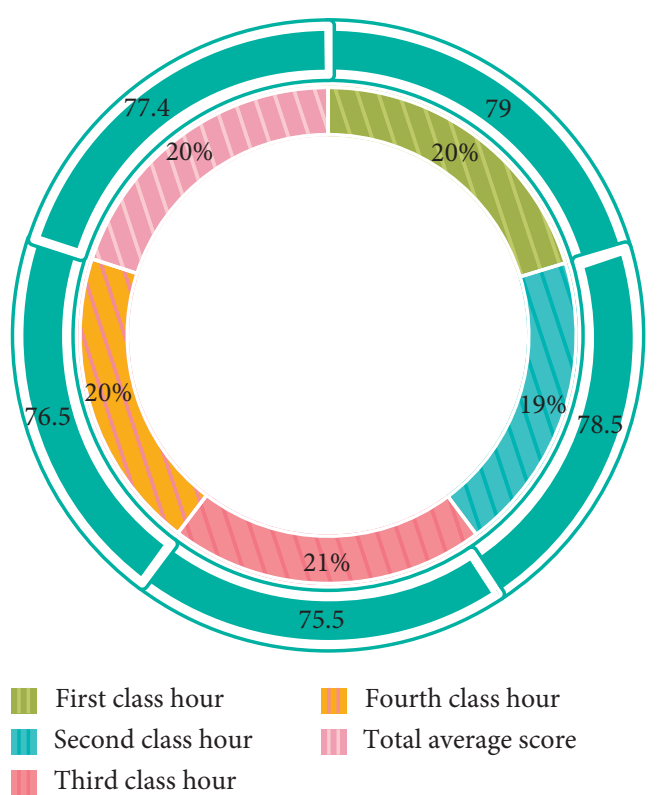

FIgURE 4: Teachers' evaluation of the swimming video teaching experiment in the class.

experimental group and the control group in the success rate of water entry, that is, $P>0.05$. The application of video image technology in the teaching of assisted swimming techniques can clearly show the student's movement process and essentials and can make the body language movements that pass by the moment change to static, turn fast to slow, and make the movements replay repeatedly. Observe at a certain point, so as to promote students' understanding and imitation and make the water entry action more standardized. Everyday's training tasks and error correction methods are targeted and purposeful. The video image technology is effectively combined with teaching and will be able to systematically solve some important and detailed problems in the technical action learning process problem. Through the gradual practice and the continuous improvement of technical movements with video image technology, the swimming movements of the experimental 
group were more standardized, which also played a certain role in promoting the students' water entry skills [26].

\section{Conclusions}

This article mainly studies the mode of video teaching of swimming sports in colleges and universities. Video feedback teaching methods have great advantages in the technical movements of more complicated or more essential sports. This teaching method can decompose the technical points very well and repeatedly present them in front of students so that students can learn about the movement technology. The mastery of the content and the mastery of the content are of great practical significance to the teaching of physical education.

The use of video feedback teaching in swimming has greatly enriched the teaching content, changed the specificity of traditional teaching, improved students' interest and desire for learning, and fully mobilized students' enthusiasm and initiative in learning so that students can gain in a short time more knowledge. Swimming education video teaching can cultivate students' cognitive thinking ability, observation, analysis, and problem-solving ability and improve classroom teaching quality, thereby improve teaching efficiency and achieve the optimization of teaching goals, teaching content, teaching process, education methods, and education effects.

The video analysis feedback training method is used during swimming training. In other words, after the athlete completes the action, he can immediately see his action video and various analysis results. In the case of sports training, video teaching can improve the training level more quickly and effectively and is effective for athletes who can shorten the time required to learn and learn technical sports. Swimming is a typical technical sport. The application of the video analysis system in swimming training helps to solve the problems of traditional education greatly. Athletes can speed up their understanding of technical sports through frequent visual stimulation and mutual image comparison. This method is especially important for the training of young students.

\section{Data Availability}

No data were used to support the findings of this study.

\section{Conflicts of Interest}

The author declares that there are no conflicts of interest.

\section{References}

[1] Y. Sakamoto, S. Okamoto, K. Shimizu, Y. Araki, A. Hirakawa, and T. Wakabayashi, "Hands-on simulation versus traditional video-learning in teaching microsurgery technique," Neurologia Medico-Chirurgica, vol. 57, no. 5, pp. 238-245, 2017.

[2] Y. Yao, "Application of flipped classroom teaching mode based on MOOC in modern educational technology teaching," Journal of Computational and Theoretical Nanoscience, vol. 14, no. 2, pp. 1075-1078, 2017.
[3] B. Peng, "Construction and application of the BEST teaching mode of college English in big data," International Journal of Emerging Technologies in Learning, vol. 12, no. 9, p. 41, 2017.

[4] Z. H. Hu, J. X. Zhou, M. J. Zhang, and Y. Zhao, "Methods for ranking college sports coaches based on data envelopment analysis and PageRank," Expert Systems, vol. 32, no. 6, pp. 652-673, 2016.

[5] D. Godish, R. Miltenberger, and S. Sanchez, "Evaluation of video modeling for teaching abduction prevention skills to children with autism spectrum disorder," Advances in Neurodevelopmental Disorders, vol. 1, no. 3, pp. 168-175, 2017.

[6] D. Shaw, "Accomplished teaching: using video recorded micro-teaching discourse to build candidate teaching competencies," Journal of Interactive Learning Research, vol. 28, no. 2, pp. 161-180, 2017.

[7] S. C. Low, A. H. Tan, and S.-Y. Lim, "Teaching video neuroImages: purposeless groaning in progressive supranuclear palsy," Neurology, vol. 88, no. 1, p. e9, 2017.

[8] P. Chauvet, R. Botchorishvili, S. Curinier et al., "What is a good teaching video? Results of an online international survey," Journal of Minimally Invasive Gynecology, vol. 27, no. 3, pp. 738-747, 2020.

[9] H. Eggink, R. Brandsma, J. H. van der Hoeven, F. Lange, T. J. de Koning, and M. A. J. Tijssen, "Teaching video neuroImages: the "round the houses" sign as a clinical clue for Niemann-Pick disease type C," Neurology, vol. 86, no. 19, p. e202, 2016.

[10] G. Rekik and N. Bali, "Initial training programs' development in physical education through video viewing courses of exemplary teaching exercises," Creative Education, vol. 8, no. 14, pp. 2199-2209, 2017.

[11] L. M. D. Santos, "English language learning for engineering students: application of a visual-only video teaching strategy," Global Journal of Engineering Education, vol. 21, no. 1, pp. 37-44, 2019.

[12] K. Kariya, T. Uchida, and H. Takahashi, "Preparation of video teaching material for human regional anatomy, especially formatted for a tablet device (iPad)," Dokkyo Journal of Medical ences, vol. 44, no. 3, pp. 227-234, 2017.

[13] L. Elfman, "Working for greater diversity and inclusion in college sports," Women in Higher Education, vol. 28, no. 1, pp. 14-15, 2019.

[14] T. O’Brien, "Rethink management, operation of college sports in light of COVID-19 pandemic," Campus Legal Advisor, vol. 20, no. 10, pp. 1-6, 2020.

[15] Y. Ono, Y. Tanaka, K. Sako, M. Tanaka, and J. Fujimoto, "Association between sports-related concussion and mouthguard use among college sports players: a case-control study based on propensity score matching," International Journal of Environmental Research and Public Health, vol. 17, no. 12 , p. $4493,2020$.

[16] M. C. Claudine, "Heed the warnings presented by the growing intersection of criminal law and college sports," College Athletics \& the Law, vol. 15, no. 3, pp. 6-7, 2018.

[17] J. T. Morton, "Invisible seasons: title IX and the fight for equity in college sports by Belanger Kelly," Journal of Sport Management, vol. 32, no. 3, pp. 1-2, 2017.

[18] J. Meyer and A. Zimbalist, "Reforming college sports: the case for a limited and conditional antitrust exemption," Antitrust Bulletin, vol. 62, no. 1, pp. 31-61, 2017.

[19] W. Yan and Y. Zhang, "College sports fitness teaching model based on constructivism," Agro Food Industry Hi-Tech, vol. 28, no. 1, pp. 1642-1645, 2017. 
[20] R. Fort, "College sports competitive balance "beliefs" and the rule of reason," Antitrust Bulletin, vol. 62, no. 1, pp. 15-30, 2017.

[21] X. Jia, "On college sports culture construction strategy based on harmonious sports," Agro Food Industry Hi-Tech, vol. 28, no. 1, pp. 3516-3519, 2017.

[22] C. McCarthy, "Learn how to reduce risks in the risky business of college sports," College Athletics and the Law, vol. 14, no. 5, p. 4, 2017.

[23] G. Hwang, "The influence of team performance on home attendance in college sports: a case study of NCAA Division I men's basketball," International Case Studies Journal, vol. 5, no. 8, pp. 187-191, 2016.

[24] H. Zhang, "Research on the influencing factors of college sports teaching based on statistical analysis," International Journal of Smart Home, vol. 10, no. 5, pp. 262-274, 2016.

[25] Z. Ting, "Research on the experiment of dynamic analysis in the college sports nutrition food courses," Advance Journal of Food Science and Technology, vol. 12, no. 2, pp. 93-96, 2016.

[26] L. Nancy, "The athletic trap: how college sports corrupted the academy by Howard L. Nixon, II," The Review of Higher Education, vol. 40, no. 1, pp. 154-157, 2016. 